\title{
Voting Power and Proportional Representation of
}

\section{Voters*}

\author{
Artyom Jelnov ${ }^{\dagger} \quad$ Yair Tauman ${ }^{\ddagger}$
}

July 3, 2012

\begin{abstract}
Our paper provides a justification for the proportional representative (PR) election system for politically diversified societies. We employ the Shapley value concept to measure the political power of parties in a parliament. We prove that for the PR system if parties' size add up to 1 and is uniformly distributed, the expected ratio of a party size to its political power converges to 1 , and the variance decreases to 0 as the number of parties increases. The rate of convergence is high. An empirical evidence from the Netherlands elections supports our result. Using the Shapley-Owen index we obtain similar result (this time numerically only) for a voting model that takes into account ideological differences between parties and voters.
\end{abstract}

${ }^{*}$ The authors thank Abraham Diskin, Pradeep Dubey, David Gilat, Dennis Leech, Abraham Neyman, Ronny Razin and Dov Samet for discussion, comments and assistance.

${ }^{\dagger}$ The Faculty of Management, Tel Aviv University, Israel.

E-mail address: artyomje@post.tau.ac.il

${ }_{\ddagger}^{\ddagger}$ Department of Economics, State University of New York, Stony Brook, NY, USA and The Interdisciplinary Center, Herzliya, Israel 


\section{Introduction}

In many democracies parliaments are elected by proportional representative system (hereafterPR). The PR system allocates seats in parliament to parties in proportion to their supporters. But does this system represent the bargaining power of the parties? The answer in general is negative. As an example, let $\mathrm{A}, \mathrm{B}$ and $\mathrm{C}$ be the only three parties represented in a parliament with 100 seats. Suppose that A, B and C have 45, 45 and 10 seats, respectively. A coalition of parties that have a simple majority (more than 50 seats) has the entire power. Any coalition of at least two parties has a majority and no party has a majority by itself. In this sense $\mathrm{C}$ has the same bargaining power as $\mathrm{A}$ or $\mathrm{B}$ even though $\mathrm{C}$ is much smaller in size. Many argue that a desired property of a 'fair' system is that "voting power" of parties should be closely related to their size. Nurmi [1981] advocates that the idea of proportional representation rests on the identity of distribution of parties' support and the distribution of parties' power. Nozick [1968, Note 4] refers to district systems and states that a system of proportional representation reflects legislators' power. The example above however demonstrates that the PR system does not satisfy this property, at least not for every distribution of parties' size. Yet, it seems that proportionality of a priori voting power to weight should be a principle on which a fair representative parliament is based. The purpose of this paper is to show that this principle is satisfied on average, when parties' size is random, and number of parties is large.

The literature offers several tools to measure voting power of a party. The most well-known tools are the Shapley value (Shapley [1953]) and the Banzhaf index (Banzhaf $[1964,1968])$. Both measures are based on the probability of party to be a pivot. Namely, the voting power of a party is the probability that it turns a random coalition of parties from one with no majority into a winning coalition. While for the Banzhaf index all coalitions have the same probability to form, the Shapley value is based on different probability distribution: coalitions of the same size are equally likely to be formed and all sizes have the same probability. 
In this paper we show that irrespective of the quota required for majority, if parties' size is uniformly distributed (reflecting no prior information about their size) the expected ratio of a party size to its voting power measured by the Shapley value converges to 1 , as the number of parties increases. This result fails to hold for the Banzhaf index. Furthermore, the rate of convergence is high and the error term is of the magnitude of $1 / n$ where $n$ is the number of parties. The variance of this ratio converges to 0 , as the number of parties increases indefinitely.

Even though the number of parties in most parliaments is relatively small our result is still applicable. A relatively small number of parties in parliaments is often caused by a "threshold of participation" (see Rae et al. [1971]). A large number of parties often participate in the election, but in some cases only small number of them succeed to obtain seats in a parliament. For instance, the 2009 German federal election resulted with 6 parties in the parliament out of 29 competing parties. The 2006 Netherlands election resulted with 10 parties out of 23 competing parties. In addition, an electoral threshold induces some parties not to participate in the elections as a distinct party.

Our result is also relevant to voting power of shareholders in a business company with relatively large number of shareholders. Since typically the number of shareholders is large our result asserts that profit sharing proportional to the number of shares reflects on average the voting power of shareholders.

The notion of voting power is well discussed in the literature. As mentioned above we focus here on the Shapley-Shubik index (Shapley and Shubik [1954]), which relies on the Shapley value solution for cooperative games (Shapley [1953]). This notion is uniquely derived by a set of four axioms and it assigns to every party in a given game a share in the total "cake". An axiomatization of the Shapley value for voting games is given in Dubey [1975]. Young [1985] provides an alternative axiomatization of the Shapley value for the class of all $n$-person games in coalitional form which can also be used to characterize the Shapley value on the class of voting games ${ }^{1}$. The Shapley value of a

\footnotetext{
${ }^{1}$ For the axiomatization of the Banzhaf index and its relationship to the Shapley value see Lehrer [1988]. Another axiomatization of the Banzhaf index is by Dubey et al. [2005]
} 
party is considered to measure its "real contribution" to the total cake, reflecting on its bargaining power in the cake division game. In the context of voting games, ShapleyShubik index measures voting power as an expected prize of a party ("the P-power", using terminology of Felsenthal and Machover $[1998]^{2}$ ). Our main result can be therefore stated as follows: if parties' size are random and has uniform distribution the expected value of the ratio of the Shapley value of a party to its size increases to 1, when the number of parties increases.

We provide an empirical evidence that supports our result. We analyze the parliamentary elections in the Netherlands. For each election we calculated the average and the variance of the ratio of the Shapley value of a party to its size. This average is above 0.9 , and the variance is impressively low.

It is worth mentioning a related and very simple result by Shapley [1961]. Namely, if the quota for majority is random and has a uniform distribution then the expected Shapley value of every party coincides with its size, irrespective of the number of parties and the specific realization of their sizes.

Finally, we use the Shapley-Owen index (see Shapley [1977]), which extends the Shapley value, to voting models where parties (and voters) have ideological differences. We use Monte-Carlo simulations to show that on average the ratio of voting power of a party to its size is close to 1 for various distributions of ideological locations of voters and parties, provided that the location of a voter is drawn from the same distribution as the location of a party.

\section{The Model}

Let $N=\{1,2, \ldots, n\}$ be the set of parties. Suppose that $X_{1}, \ldots, X_{n}$ are $n$ random variables that measure the size of the $n$ parties. That is

\footnotetext{
${ }^{2}$ Although Felsenthal and Machover [1998] criticizes the Shapley-Shubik index and even the idea of P-power, in Felsenthal and Machover [2005] they state that "for a priori P-power, the Shapley-Shubik index still seems to be the most reasonable candidate for measuring it "
} 
$\sum_{i=1}^{n} X_{i}=1, X_{i} \geq 0$ and $i=1, \ldots, n$. Let

$$
A_{n}=\left\{\left(x_{1}, \ldots, x_{n}\right) \mid \sum_{i=1}^{n} x_{n}=1, x_{i} \geq 0, i=1, \ldots, n\right\}
$$

be the $n-1$ dimensional simplex in $\mathbb{R}^{n}$. We assume that the realization $\left(x_{1}, \ldots, x_{n}\right)$ has a uniform distribution on $A_{n}$ with respect to the volume of $A_{n}$. Let $v_{n}$ be the volume of $A_{n}$ and let $p_{n}=\frac{1}{v_{n}}$ be the (fixed) density function of $X=\left(X_{1}, \ldots, X_{n}\right)$ on $A_{n}$.

Let $\frac{1}{2} \leq q<1$ be a quota and let $V_{n}$ be the voting game on $N$ defined for every realization $x \in A_{n}$ and all $S \subseteq N$ by

$$
V_{n}(S, x)= \begin{cases}1 & , \sum_{i \in S} x_{i}>q \\ 0 & , \text { otherwise }\end{cases}
$$

We say that a subset $S$ of $N$ is a winning coalition if $\sum_{i \in S} x_{i}>q$ and it is a minimal winning coalition if it is a winning coalition and for all $i \in S S \backslash\{i\}$ is not a winning coalition $\left(\sum_{j \in S \backslash\{i\}} x_{j} \leq q\right)$.

Let $x \in A_{n}$ and let $\Theta_{i}(x)$ be the set of all coalitions $S, S \subseteq N \backslash\{i\}$, such that $S \cup\{i\}$ is a minimal winning coalition. In this case we say that $i$ is a pivot player to $S$. That is, $\Theta_{i}(x)$ is the set of all coalitions $S, S \subseteq N \backslash\{i\}$ such that $i$ is pivot to $S$.

To derive the Shapley value of a player consider the $n$ ! permutations of the players in $N$. For every $i \in N$ and every permutation $\Re$ let $P_{i}^{\Re}$ be the subset of players in $N$ that precede $i$ in the order $\Re$. For example, suppose that $N=\{1,2,3,4\}$ and $\Re=\{2,3,1,4\}$. Then $P_{1}^{\Re}=\{2,3\}$.

The number of permutations of $N$ where $i$ is a pivot is

$$
\phi(x, i)=\sum_{S \in \Theta_{i}(x)}|S| !(n-|S|-1) !
$$

Given the set $N$ and the weights $x=\left(x_{1}, \ldots, x_{n}\right) \in A_{n}$, the Shapley value of $V_{n}$ is

$$
S h_{i}(x)=\frac{\phi(x, i)}{n !}
$$

That is, the Shapley value of a party $i$ is the probability that $i$ is a pivot in a random order where all orders are equally likely. An equivalent way to derive the Shapley value of $i \in N$ is through the following probability distribution over coalitions $S \subseteq N \backslash\{i\}$. All 
coalitions of the same size are equally likely to be formed and all sizes $0,1, \ldots, n-1$ have the same probability, $\frac{1}{n}$. That is the probability of $S \subseteq N \backslash\{i\}$ is $\frac{1}{\left(\begin{array}{c}n-1 \\ |S|\end{array}\right) n}=\frac{|S|(n-|S|-1)}{n !}$.

Given $(N, x)$ the Banzhaf index, $B z$, of $i \in N$ is:

$$
B z_{i}(x)=\frac{\left|\Theta_{i}(x)\right|}{2^{n-1}}
$$

That is, every coalition $S \subseteq N \backslash\{i\}$ have the same probability to form irrespective of its size.

Thus the Shapley value and the Banzhaf index of a party $i$ are both probability of $i$ to be a pivot to a random coalition. The two measures differ in the probability distribution over coalitions.

Let Exp be the expected value operator and denote

$$
\operatorname{Exp}\left(S h_{i}(X), n\right)=\int_{A_{n}} p_{n} S h_{i}(X) d X
$$

and

$$
\operatorname{Exp}\left(\frac{S h_{i}(X)}{X_{i}}, n\right)=\int_{A_{n}} p_{n} \frac{S h_{i}(X)}{X_{i}} d X
$$

Similarly,

$$
\operatorname{Exp}\left(\frac{B z_{i}(X)}{X_{i}}, n\right)=\int_{A_{n}} p_{n} \frac{B z_{i}(X)}{X_{i}} d X .
$$

The following result is shown analytically.

Theorem For every $i \in N$, and $\frac{1}{2} \leq q<1$

(1) $\operatorname{Exp}\left(\frac{S h_{i}(X)}{X_{i}}, n\right)$ is increasing in $n$ and

$$
\lim _{n \rightarrow \infty} \operatorname{Exp}\left(\frac{S h_{i}(X)}{X_{i}}, n\right)=1
$$

(2) $\operatorname{Exp}\left(\frac{S h_{i}(X)}{X_{i}}, n\right)=1+O\left(\frac{1}{n}\right)$

That is, the expected ratio between the Shapley value and the size of a party increases to 1 as $n$ increases indefinitely. The rate of convergence is $\frac{1}{n}$ and it can be shown that the error term converges to zero exponentially. Figure 1 illustrates the rate of convergence for $q=1 / 2$. In this case, $\operatorname{Exp}\left(\frac{S h_{i}(X)}{X_{i}}, n\right) \geq 0.9$ for $n \geq 10$. 


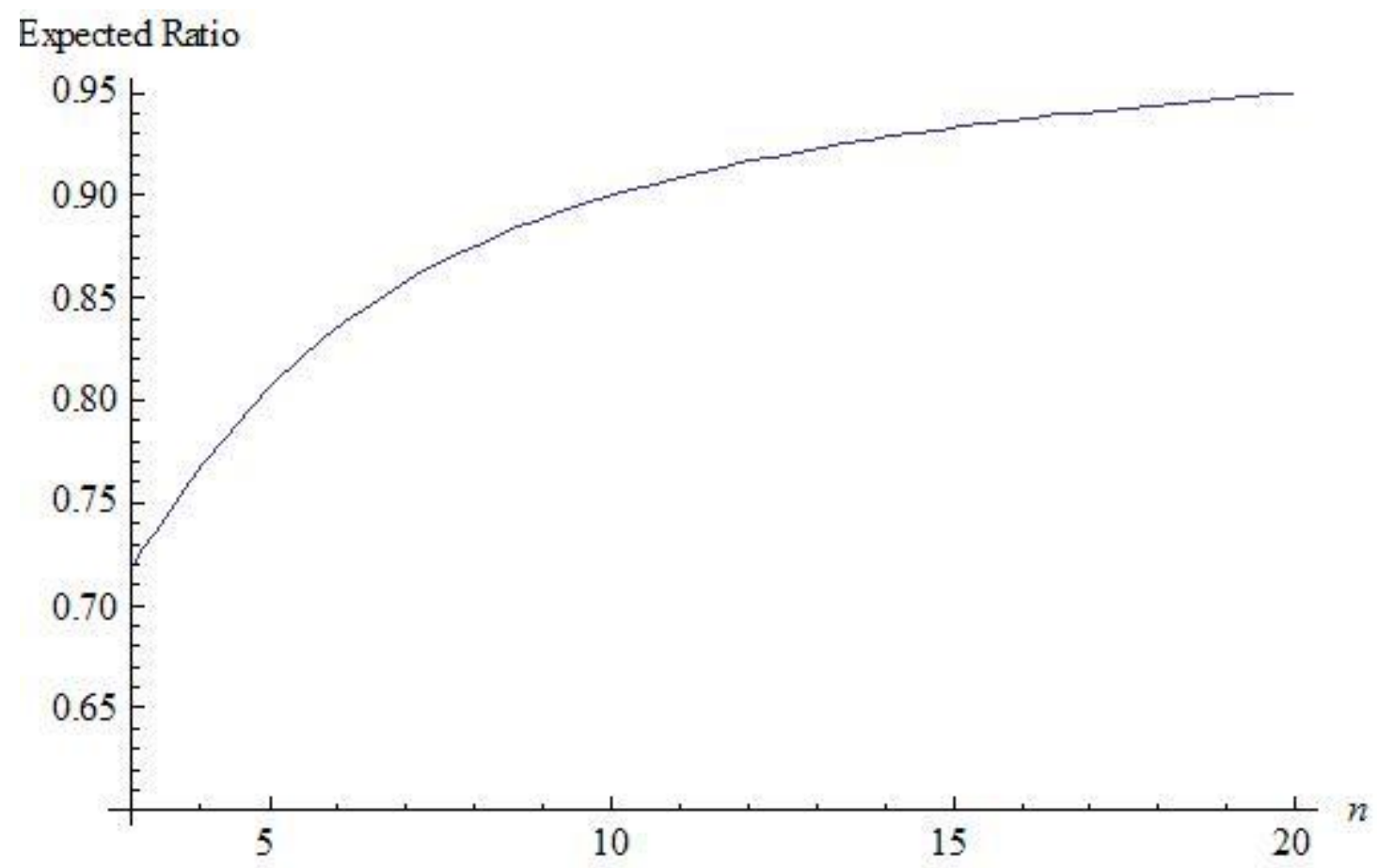

Figure 1: $\operatorname{Exp}\left(\frac{S h_{i}(X)}{X_{i}}, n\right)$ for $q=0.5$

\section{Example}

1. Suppose that $n=2$ (two parties only). Then $X_{i} \sim U[0,1], i=1,2$. Clearly

$$
S h_{i}(x)= \begin{cases}1 & , x_{i}>q \\ 0 & , x_{i} \leq q\end{cases}
$$

implying that

$$
\operatorname{Exp}\left(\frac{S h_{i}(X)}{X_{i}}, 2\right)=\int_{q}^{1} \frac{1}{x_{i}} d x_{i}=-\log q .
$$

In particular for $q=\frac{1}{2} \operatorname{Exp}\left(\frac{S_{i}(X)}{X_{i}}, 2\right)=\log 2<1$

2. Suppose next that $n=3$. The computation of $\operatorname{Exp}\left(\frac{S h_{i}(X)}{X_{i}}, 3\right)$ is more complicated.

We show later on (see (6) below) that for $q=\frac{1}{2}$

$$
\operatorname{Exp}\left(\frac{S h_{i}(X)}{X_{i}}, 3\right)=2 \log 2-\frac{2}{3}
$$

and $\log 2<2 \log 2-\frac{2}{3}<1^{3}$.

\footnotetext{
${ }^{3}$ We provide an explicit computation of $\operatorname{Exp}\left(\frac{S h_{i}(X)}{X_{i}}, n\right)$ for all $q, \frac{1}{2} \leq q<1$ and all $n$.
} 
To prove the Theorem we first state and prove the following proposition.

Let $c \in(0,1)$ and let $C_{n}(c)$ be the set of all elements in $A_{n}$ such that $x_{1}=c$.

$$
C_{n}(c)=\left\{\left(c, x_{2}, \ldots, x_{n}\right) \mid \sum_{i=2}^{n} x_{i}=1-c, x_{i} \geq 0, i=2, \ldots, n\right\} .
$$

Proposition 1 Suppose that the elements of $C_{n}(c)$ are uniformly distributed. Then for $n \geq 3$

$$
\int_{C_{n}(c)} p_{n}^{\prime} S h_{1}(X) d X= \begin{cases}\frac{c(n-2)}{(1-c) n} & , 0<c<1-q \\ \frac{1}{n}+\frac{(n-2)(1-q)}{n(1-c)} & , 1-q \leq c \leq q \\ 1 & , q<c \leq 1,\end{cases}
$$

where $p_{n}^{\prime}$ is the (fixed) density function of $X=\left(X_{2}, \ldots, X_{n}\right)$ on $C_{n}(c)$.

Note, that Proposition 1 is consistent with the well-known "oceanic games" result (Shapiro and Shapley [1978]), which states, that if there is a sequence of weighted majority games with one party of constant size $c<q$ (a major party), and the size of any other (minor) party converges to zero, then the Shapley value of the major party converges to $\frac{c}{1-c}$. It was shown in Dubey and Shapley [1979] that the convergence of the Banzhaf index is different.

The proof of the Proposition relies on the following two well-known lemmas.

Lemma 1 Let $Y_{1}, \ldots, Y_{n}$ be i.i.d. with exponential distribution. Then $\left(X_{1}, \ldots, X_{n}\right)$ and $\left(\frac{Y_{1}}{\sum_{j=1}^{n} Y_{j}}, \ldots, \frac{Y_{n}}{\sum_{j=1}^{n} Y_{j}}\right)$ has the same distribution.

For a proof see Feller [1971].

Lemma 2 Let $Y_{1}, \ldots, Y_{n}$ be i.i.d. random variables, each has an exponential distribution. For $1 \leq k \leq n$, let $\Sigma_{k}=\sum_{i=1}^{k} Y_{i}$. Then $\frac{\Sigma_{k}}{\Sigma_{n}}$ for $1 \leq k<n$ has the Beta distribution with parameters $(k, n-k)$.

For a proof see Jambunathan [1954], Theorem 3.

Notice that the Beta distribution function is defined by

$$
P\left(\frac{\Sigma_{k}}{\Sigma_{n}}<z\right)=\sum_{j=k}^{n-1}\left(\begin{array}{c}
n-1 \\
j
\end{array}\right) z^{j}(1-z)^{n-1-j}
$$

The next lemma is a consequence of the above two lemmas. 
Lemma 3 Suppose that $X=\left(X_{1}, \ldots, X_{m}\right)$ is uniformly distributed on $A_{m}$, where $m \geq 2$. Then

$$
\sum_{k=1}^{m-1} P\left(\sum_{i=1}^{k} X_{i} \leq z\right)=(m-1) z
$$

\section{Proof}

From Lemmas 1 and $2, \sum_{i=1}^{k} X_{i}$ has Beta distribution with parameters $(k, m-k)$. Hence,

$$
\sum_{k=1}^{m-1} P\left(\sum_{i=1}^{k} X_{i} \leq z\right)=\sum_{k=1}^{m-1} \sum_{j=k}^{m-1} \frac{(m-1) !}{j !(m-1-j) !} z^{j}(1-z)^{m-1-j}
$$

By rearranging terms we have:

$$
\begin{aligned}
\sum_{k=1}^{m-1} P\left(\sum_{i=1}^{k} X_{i} \leq z\right) & =\sum_{k=1}^{m-1} k \frac{(m-1) !}{k !(m-1-k) !} z^{k}(1-z)^{m-1-k}= \\
& =(m-1) z \sum_{k=1}^{m-1}\left(\begin{array}{c}
m-2 \\
k-1
\end{array}\right) z^{k-1}(1-z)^{m-1-k}= \\
& =(m-1) z \sum_{k^{\prime}=0}^{m-2}\left(\begin{array}{c}
m-2 \\
k^{\prime}
\end{array}\right) z^{k^{\prime}}(1-z)^{m-2-k^{\prime}}= \\
& =z(m-1)(z+1-z)^{m-2}=z(m-1) \square
\end{aligned}
$$

Corollary 1 Suppose that $X$ is uniformly distributed on $C_{m}(c), m \geq 3$. Then

$$
\sum_{k=2}^{m-1} P\left(\sum_{i=2}^{k} X_{i} \leq z\right)=(m-2) \frac{z}{1-c}
$$

We are ready now to prove Proposition 1.

\section{Proof of Proposition 1}

For every permutation $\Re$ of $N$ party 1 is pivot if $q-c<\sum_{i \in P_{i}^{\Re}} x_{i} \leq q$. Denote by $\Phi_{\Re}=\left\{\left(c, x_{2}, \ldots, x_{n}\right) \in C_{n}(c) \mid q-c<\sum_{i \in P_{i}^{\Re}} x_{i} \leq q\right\}$ the subset of $C_{n}(c)$, in which party 1 is pivot in $\Re$.

Let $\Re_{k}$ be the set of all orders $\Re$ of $N$ such that there are exactly $k$ parties that precede 1 in the order $\Re$. Note that $C_{n}(c)$ is a symmetric subset of $\mathbb{R}^{n}$ and so is $\Phi_{\Re}$ for every order $\Re$. Hence, if $\Re \in R_{k}$ and $\Re^{\prime} \in R_{k}$

$$
P\left(X \in \Phi_{\Re} \mid X \in C_{n}(c)\right)=P\left(X \in \Phi_{\Re^{\prime}} \mid X \in C_{n}(c)\right) \equiv \Pi(c, k)
$$


Since the orders of $N$ are uniformly distributed, $\operatorname{Prob}\left(\Re \in R_{k}\right)=\frac{1}{n}$. Thus

$$
\int_{C_{n}(c)} p_{n}^{\prime} S h_{1}(X) d X=\frac{1}{n} \sum_{k=0}^{n-1} \Pi(c, k)=\frac{1}{n} \sum_{k=1}^{n-1} \Pi(c, k)
$$

Notice that

$$
\Pi(c, k)=P\left(q-c<\sum_{i=2}^{k+1} X_{i} \leq q\right)
$$

We distinguish two cases.

Case $10<c<1-q$

In this case $\Pi(c, 0)=\Pi(c, n-1)=0$ and by $(2)$

$\frac{1}{n} \sum_{k=1}^{n-2} \Pi(c, k)=\frac{1}{n} \sum_{k=1}^{n-2} P\left(q-c<\sum_{i=2}^{k+1} X_{i} \leq q\right)=\frac{1}{n}\left[\sum_{k=1}^{n-2} P\left(\sum_{i=2}^{k+1} X_{i} \leq q\right)-\sum_{k=1}^{n-2} P\left(\sum_{i=2}^{k+1} X_{i} \leq q-c\right)\right]$

By Corollary 1

$$
\frac{1}{n} \sum_{k=1}^{n-2} \Pi(c, k)=\frac{n-2}{n(1-c)}[q-(q-c)]=\frac{n-2}{n} \frac{c}{1-c}
$$

This together with (1) imply

$$
\int_{C_{n}(c)} p_{n}^{\prime} S h_{1}(X) d X=\frac{n-2}{n} \frac{c}{1-c}
$$

as claimed.

Case $21-q \leq c \leq q$

In this case party 1 is a veto player meaning that every winning coalition must include 1. In this case $P\left(\sum_{i=2}^{k+1} X_{i} \leq q\right)=1$ for every $k=1, \ldots, n-1$, and in particular $\Pi(c, n-1)=1$. Applying (2) we have

$$
\frac{1}{n} \sum_{k=1}^{n-1} \Pi(c, k)=\frac{1}{n}+\frac{1}{n} \sum_{k=1}^{n-2} \Pi(c, k)=\frac{1}{n}+\frac{n-2}{n}-\frac{1}{n} \sum_{k=1}^{n-2} P\left(\sum_{i=2}^{k+1} X_{i} \leq q-c\right) .
$$

By Corollary 1

$$
\frac{1}{n} \sum_{k=1}^{n-1} \Pi(c, k)=\frac{1}{n}+\frac{n-2}{n}-\frac{(n-2)(q-c)}{n(1-c)}=\frac{1}{n}+\frac{(n-2)(1-q)}{n(1-c)}
$$

By (1) and (3)

$$
\int_{C_{n}(c)} p_{n}^{\prime} S h_{1}(X) d X=\frac{1}{n}+\frac{(n-2)(1-q)}{n(1-c)}
$$


Note that if $c>q$ then 1 is dictator and is a pivot in every order $\Re$. In this case its Shapley value is 1 .

We are now ready to prove the theorem.

Proof of the Theorem Without loss of generality we prove the theorem for $i=1$. Let $f_{X_{i}}\left(x_{i}\right)$ be the density distribution function of $X_{i}$ (derived from the fact that $X=$ $\left(X_{1}, \ldots, X_{n}\right)$ has a uniform distribution on $A_{n}$.

Lemma $4 f_{X_{i}}(x)=(n-1)(1-x)^{n-2}$

Proof By Lemmas 1 and 2

$$
\begin{gathered}
X_{i} \sim \frac{Y_{i}}{\sum_{j=1}^{n} Y_{j}} \sim \beta(1, n-1) \\
F_{X_{i}}(x)=P\left(X_{i} \leq x\right)=\sum_{j=1}^{n-1}\left(\begin{array}{c}
n-1 \\
j
\end{array}\right) x^{j}(1-x)^{n-1-j}= \\
=\sum_{j=0}^{n-1}\left(\begin{array}{c}
n-1 \\
j
\end{array}\right) x^{j}(1-x)^{n-1-j}-(1-x)^{n-1}=1-(1-x)^{n-1}
\end{gathered}
$$

Consequently

$$
f_{X_{i}}(x)=(n-1)(1-x)^{n-2}
$$

as claimed.

Next define for every $x_{1}, 0 \leq x_{1} \leq 1$, the set $B_{n-1}\left(x_{1}\right) \subseteq \mathbb{R}^{n-1}$ by

$$
B_{n-1}\left(x_{1}\right)=\left\{\left(x_{1}, \ldots, x_{n}\right) \mid \sum_{j=2}^{n} x_{j}=1-x_{1}\right\}
$$

Also denote by $f_{X_{-1}}\left(x_{2}, \ldots, x_{n} \mid X_{1}=x_{1}\right)$ the conditional density function of $\left(X_{2}, \ldots, X_{n}\right)$ on $B_{n-1}\left(x_{1}\right)$. Then

$$
\begin{aligned}
\operatorname{Exp}\left(\frac{S h_{1}(X)}{X_{1}}, n\right) & =\int_{x \in A_{n}} p_{n} \frac{S h_{1}(x)}{x_{1}} d x_{1}, \ldots, d x_{n}= \\
& =\int_{0}^{1} f_{X_{1}}\left(x_{1}\right)\left[\int_{B_{n-1}\left(x_{1}\right)} f_{X_{-1}}\left(x_{2}, \ldots, x_{n} \mid x_{1}\right) \frac{S h_{1}(x)}{x_{1}} d x_{2}, \ldots, d x_{n}\right] d x_{1}=
\end{aligned}
$$

By Lemma 4

$$
=\int_{0}^{1}(n-1)\left(1-x_{1}\right)^{n-2}\left[\int_{B_{n-1}\left(x_{1}\right)} f_{X_{-1}}\left(x_{2}, \ldots, x_{n} \mid x_{1}\right) \frac{S h_{1}(x)}{x_{1}} d x_{2}, \ldots, d x_{n}\right] d x_{1}
$$


Note that $S h_{1}(x)=1$ whenever $x_{1}>q(1$ is a dictator in this case). Also, by Proposition 1 if $x_{1} \leq q$ then

$$
\begin{aligned}
& \int_{B_{n-1}\left(x_{1}\right)} f_{X_{-1}}\left(x_{2}, \ldots, x_{n} \mid x_{1}\right) S h_{1}(x) d x_{2}, \ldots, d x_{n}=\int_{C_{n}\left(x_{1}\right)} p_{n}^{\prime} S h_{1}(x) d x= \\
& = \begin{cases}\frac{n-2}{n} \frac{x_{1}}{1-x_{1}} & , x_{1} \leq 1-q \\
\frac{1}{n}+\frac{(n-2)}{n\left(1-x_{1}\right)}(1-q) & , 1-q<x_{1} \leq q\end{cases}
\end{aligned}
$$

By (4) and (5)

$$
\begin{aligned}
\operatorname{Exp}\left(\frac{S h_{1}(X)}{X_{1}}, n\right) & =\int_{0}^{1-q} \frac{(n-1)(n-2)}{n}\left(1-x_{1}\right)^{n-3} d x_{1}+ \\
& +\int_{1-q}^{q}\left[\frac{(n-1)}{n} \frac{\left(1-x_{1}\right)^{n-2}}{x_{1}}+\right. \\
& \left.+\frac{(n-1)(n-2)}{n} \frac{\left(1-x_{1}\right)^{n-3}(1-q)}{x_{1}}\right] d x_{1}+ \\
& +\int_{q}^{1} \frac{(n-1)\left(1-x_{1}\right)^{n-2}}{x_{1}} d x_{1}
\end{aligned}
$$

But

$$
\int_{0}^{1-q} \frac{(n-1)(n-2)}{n}\left(1-x_{1}\right)^{n-3} d x_{1}=-\left.\frac{n-1}{n}\left(1-x_{1}\right)^{n-2}\right|_{0} ^{1-q}=\frac{n-1}{n}-\frac{n-1}{n} q^{n-2}
$$

and

$$
\begin{aligned}
0 & \leq \int_{1-q}^{q}\left[\frac{(n-1)}{n} \frac{\left(1-x_{1}\right)^{n-2}}{x_{1}}+\frac{(n-1)(n-2)}{n} \frac{\left(1-x_{1}\right)^{n-3}(1-q)}{x_{1}}\right] d x_{1} \leq \\
& \leq \frac{n-1}{n(1-q)} \int_{1-q}^{q}\left[\left(1-x_{1}\right)^{n-2}+(n-2)\left(1-x_{1}\right)^{n-3}(1-q)\right] d x_{1}= \\
& =\left.\frac{n-1}{n(1-q)}\left[-\frac{\left(1-x_{1}\right)^{n-1}}{n-1}-(1-q)\left(1-x_{1}\right)^{n-2}\right]\right|_{1-q} ^{q}= \\
& =\frac{q^{n-1}}{n(1-q)}+\frac{(n-1) q^{n-2}}{n}-\frac{(1-q)^{n-2}}{n}-\frac{(1-q)^{n-2}(n-1)}{n}
\end{aligned}
$$

and

$$
\begin{aligned}
0 & \leq \int_{q}^{1} \frac{(n-1)\left(1-x_{1}\right)^{n-2}}{x_{1}} d x_{1} \leq \frac{n-1}{q} \int_{q}^{1}\left(1-x_{1}\right)^{n-2} d x_{1}= \\
& =-\left.\frac{1}{q}\left(1-x_{1}\right)^{n-1}\right|_{q} ^{1}=\frac{(1-q)^{n-1}}{q}
\end{aligned}
$$

Consequently by (6),(7),(8) and (9)

$$
\frac{n-1}{n}\left(1-q^{n-2}\right) \leq \operatorname{Exp}\left(\frac{S h_{1}(X)}{X_{1}}, n\right) \leq \frac{n-1}{n}+\frac{q^{n-1}}{n(1-q)}-(1-q)^{n-2}+\frac{(1-q)^{n-1}}{q}
$$


Equivalently

$$
-\frac{1}{n}-\frac{n-1}{n} q^{n-2} \leq \operatorname{Exp}\left(\frac{S h_{1}(X)}{X_{1}}, n\right)-1 \leq \frac{1}{n}+\frac{q^{n-1}}{n(1-q)}-(1-q)^{n-2}+\frac{(1-q)^{n-1}}{q} .
$$

Since $0<q<1$ for every $l>0$

$$
\operatorname{Exp}\left(\frac{S h_{1}(X)}{X_{1}}, n\right)-1=O\left(\frac{1}{n}\right)
$$

Using (6) it is straightforward to show that $\operatorname{Exp}\left(\frac{S h_{1}(X)}{X_{1}}, n\right)$ increases in $n$, and the proof of the theorem is complete.

Remark 1 The assumption that $A_{n}$ has uniform distribution is essential. The result does not hold in general. As a trivial example, suppose that the distribution on $A_{n}$ is such that $P\left(q<X_{1} \leq 1-\epsilon\right)=1,0<\epsilon<1-q$. In this case $S h_{1}(X)=1$ and $X_{1}<1$ with probability 1 . In addition, it is obvious from Proposition 1 , that for $q=0.5$ if the size of Party 1 is $c$ with probability 1 , and size of other parties are distributed uniformly, then for $n$ sufficiently large $\operatorname{Exp}\left(\frac{S h_{1}(X)}{X_{1}}, n\right)$ converges to $\frac{1}{1-c}$, which is different from 1 .

Remark 2 The variance of the random ratio $\frac{S h_{i}(X)}{X_{i}}$ can be calculated numerically using Monte-Carlo simulation and the approximation method of Owen [1975]. The simulation shows, that for $q=0.5$ the variance of $\frac{S h_{i}(X)}{X_{i}}$ is small and converges to 0 when $n$ increases ( see Figure 2). 


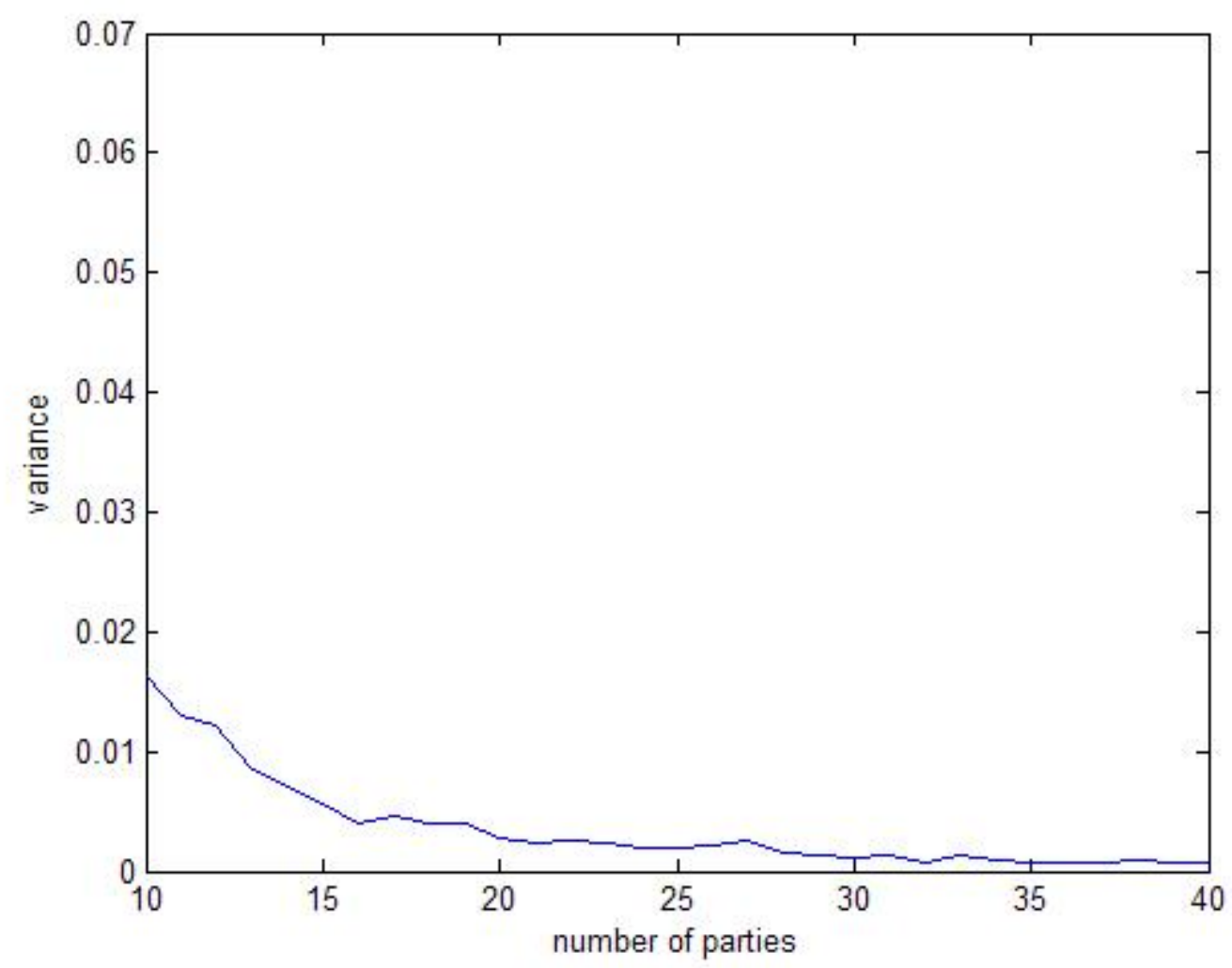

Figure 2: $\operatorname{Var}\left(\frac{S h_{i}(X)}{X_{i}}, n\right), q=0.5$

\section{The Banzhaf index}

It is natural to ask whether a result similar to the one stated in the Theorem holds for the Banzhaf index, namely, whether $\lim _{n \rightarrow \infty} \operatorname{Exp}\left(\frac{B z_{1}(X)}{X_{1}}, n\right)=1$ for all $q \geq 0.5$. As we will show below, this statement is false.

Lemma 5 Let $q=0.5$. Then

$$
\begin{aligned}
& \operatorname{Exp}\left(\frac{B z_{1}(X)}{X_{1}}, n\right)= \\
& =\frac{1}{2^{n-1}} \int_{0}^{0.5}(1-n)\left(1-x_{i}\right)^{n-2}\left[\sum_{k=1}^{n-2}\left(\begin{array}{c}
n-1 \\
k
\end{array}\right) \sum_{j=k}^{n-2}\left(\left(\begin{array}{c}
n-2 \\
j
\end{array}\right)\left[\left(\frac{0.5}{1-x_{1}}\right)^{j}\left(1-\frac{0.5}{1-x_{1}}\right)^{n-2-j}\right]\right)-\right. \\
& \left.\left.\left.-\left(\frac{0.5-x_{1}}{1-x_{1}}\right)^{j}\left(1-\frac{0.5-x_{1}}{1-x_{1}}\right)^{n-2-j}\right]\right)\right] \frac{1}{x_{1}} d x+ \\
& +\int_{0.5}^{1} \frac{(1-n)\left(1-x_{1}\right)^{n-2}}{x_{1}} d x_{1}
\end{aligned}
$$


Proof The proof is similar to the proof of the Theorem.

Note that if $c>0.5, B z_{i}(X)=1$. Suppose $c \leq q$.

Let $S \subseteq N,|S|=k$. Denote

$$
\Pi(c, k)=P\left(q-c<\sum_{i \in S} X_{i} \leq q\right)
$$

From Lemmas 1 and 2,

$$
\begin{aligned}
P\left(q-c<\sum_{i \in S} X_{i} \leq q\right) & =\sum_{j=k}^{n-2}\left(\left(\begin{array}{c}
n-2 \\
j
\end{array}\right)\left[\left(\frac{0.5}{1-x_{1}}\right)^{j}\left(1-\frac{0.5}{1-x_{1}}\right)^{n-2-j}\right]\right)- \\
& \left.\left.-\left(\frac{0.5-x_{1}}{1-x_{1}}\right)^{j}\left(1-\frac{0.5-x_{1}}{1-x_{1}}\right)^{n-2-j}\right]\right)
\end{aligned}
$$

The probability that a coalition of size $k$ is formed is $\left(\begin{array}{c}n-1 \\ k\end{array}\right) \frac{1}{2^{n-1}}$

Similar to (4),

$$
\begin{aligned}
\operatorname{Exp}\left(\frac{B z_{1}(X)}{X_{1}}, n\right) & =\int_{0}^{1}(n-1)\left(1-x_{1}\right)^{n-2}\left[\int_{B_{n-1}\left(x_{1}\right)} f_{X_{-1}}\left(x_{2}, \ldots, x_{n} \mid x_{1}\right) \frac{B z_{1}(x)}{x_{1}} d x_{2}, \ldots, d x_{n}\right] d x_{1}= \\
& =\int_{0}^{0.5} \frac{(n-1)\left(1-x_{1}\right)^{n-2}}{x_{1}} \sum_{k=1}^{n-2} \frac{1}{2^{n-1}} \Pi(c, k) d x_{1}+ \\
& +\int_{0.5}^{1} \frac{(n-1)\left(1-x_{1}\right)^{n-2}}{x_{1}} d x_{1}
\end{aligned}
$$

The lemma now follows by (11) and (12).

Figure 3 below describes the numerical calculation of $\operatorname{Exp}\left(\frac{B z_{1}(X)}{X_{1}}, n\right)$ using Lemma 5 above. It illustrates that $\operatorname{Exp}\left(\frac{B z(X)}{X_{1}}, n\right)$ does not converge to 1 . 


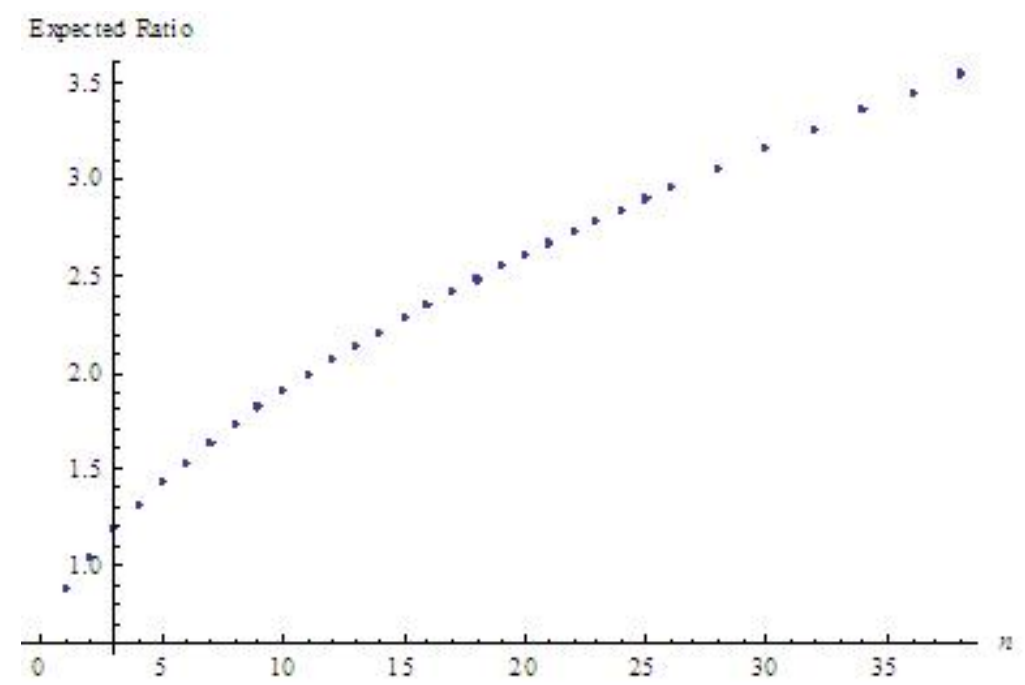

Figure 3: $\operatorname{Exp}\left(\frac{B z_{i}(X)}{X_{i}}, n\right)$ for $q=0.5$

\section{Empirical evidence}

Consider the elections of the Second Chamber ("Tweede Kamer") of the Netherlands' parliament. We analyze all elections since 1918 (the first time when the PR system was introduced in the Netherlands). The data was taken from Mackie and Rose [1991], Van Der Eijk [1989],Lucardie and Voerman [1995],Irwin [1999],Lucardie [2003],Lucardie and Voerman [2004],Lucardie [2007] and Lucardie and Voerman [2011]. In these elections we only consider parties that entered the parliament ${ }^{4}$. For each party we calculate the ratio of its Shapley value to its size (when the size is defined as the fraction of popular vote it received). For each election we calculate the expected value and the variance of this ratio.

When the number of parties is 10 or more, we use the approximation method of Owen

\footnotetext{
${ }^{4}$ We also ignored parties classified as "others" in the data sources we used. In most cases those parties did not obtain sufficient votes to pass the electoral threshold
} 
[1975] to calculate the Shapley value.
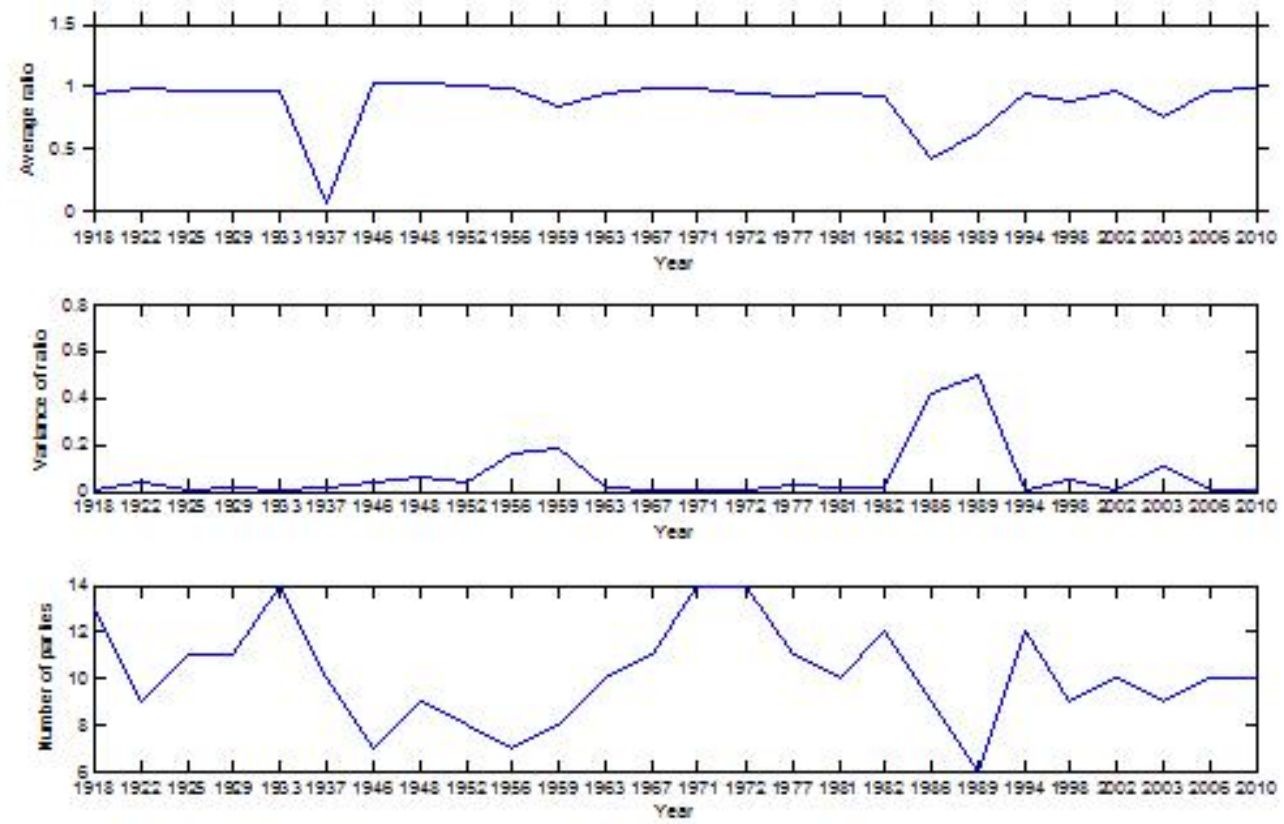

Figure 4: The data analysis for the elections in the Netherlands, 1918-2010

When the number of parties is large than 9 , the average ratio is close to 1 , and the variance is close to 0 .

Remark 3 The same calculation could be done for the Banzhaf index. It can be shown, that in most cases the expected value of the ratio of the party's Banzhaf index to its size is close to be constant, but the variance is relatively high.

\section{Voting power on ideological space}

The Shapley-Shubik index measures the probability that a party will be pivotal to a random coalition. This ignores any ideological difference of voters and parties. To deal with ideological proximity of parties and voters it is assumed that parties and voters are located in an ideological space, $D$, which is a subset of $\mathbb{R}^{d}$, where $d$ is the number 
of factors or attributes that determines voters' positions. Every point in $D$ represents a voter. The (Euclidean) distance between any two points in $D$ represents ideological differences of voters (and parties). The assumption is that every voter votes for the party that has the smallest proximity to his/her location in $D$. This is in the spirit of the wellknown Hotelling model ( Hotelling [1929]). Owen [1971] and Shapley [1977] proposed an adaptation of the Shapley-Shubik index to such spatially placed voters (see also Straffin [1994] for a survey of this literature).

The locations of the parties are random variables $Y=\left(Y_{1}, \ldots, Y_{n}\right)$ and their realization is $y=\left(y_{1}, \ldots, y_{n}\right) \in D^{n}$. Voters are distributed on $D$ according to a continuous density distribution function $f: D \rightarrow \mathbb{R}_{+}^{d}$ where $\int_{D} f(z) d z=1$.

It is assumed that $Y_{1}, \ldots, Y_{n}$ are i.i.d. and each $Y_{i}$ has the same distribution as the voters location.

For every party $i \in N$ and every location $\left(y_{1}, \ldots, y_{n}\right)$ of the parties the set of voters that vote for $i$ is

$$
L_{i}=\left\{z \mid \sum_{j=1}^{d}\left(z_{j}-y_{i, j}\right)^{2} \leq \sum_{j=1}^{n}\left(z_{j}-y_{k, j}\right)^{2}, \forall k \in N\right\}
$$

That is, $L_{i}$ is the set of all voters that are closer to party $i$ than to any other party (the measure of voters that are equally close to two or more parties with different locations is zero). The size of Party $i$ is

$$
x_{i}(y)=\int_{L_{i}} f(z) d z
$$

and clearly $\sum_{i=1}^{n} x_{i}(y)=1$.

We next introduce the Shapley-Owen index (Shapley [1977]) to measure the voting power of parties.

Let $\Omega$ be the $d$-dimensional unit ball. Namely

$$
\Omega=\left\{\omega \in \mathbb{R}^{d} \mid \sum_{j=1}^{d} \omega_{j}^{2}=1\right\}
$$

Every $\omega \in \Omega$ describes an "ideological direction" or a specific mix of ideological factors. All directions are assumed to be equally likely. Namely, the distribution over elements in $\Omega$ is uniform. 
Let $f_{\Omega}$ be the uniform density distribution over $\Omega$. Each direction $\omega \in \Omega$ defines a particular order of the parties (described formally below) representing one dimensional proximity of parties with respect to $\omega$. The distribution over $\Omega$ induces probability distribution over the different orders of parties and hence a probability of a party to be pivotal. Let us next define formally the order of parties induced by $\omega \in \Omega$, given their locations $y_{1}, \ldots, y_{n}$ in $\mathbb{R}^{d}$.

Each $\omega \in \Omega$ defines a preference relation $\prec_{\omega}$ on $D$ over the location of the parties as follows:

$$
y_{i} \prec_{\omega} y_{k} \text { iff either } \sum_{j}^{d} y_{i, j} \omega_{j}<\sum_{j}^{d} y_{k, j} \omega_{j} \text { or if } i<k \text { and } \sum_{j}^{d} y_{i, j} \omega_{j}=\sum_{j}^{d} y_{k, j} \omega_{j}
$$

Geometrically, $y_{i} \prec_{\omega} y_{k}$ iff the projection of $y_{i}$ on $\vec{\omega}$ exceeds the projection of $y_{j}$ on $\vec{\omega}$ in the direction of $\vec{\omega}$.

Given $y \in \mathbb{R}^{d n}, \prec_{\omega}$ defines a permutation $\Re_{\omega, y}$ on $N$ by

$$
\Re_{\omega, y}(i)<\Re_{\omega, y}(k) \text { iff } y_{i} \prec_{\omega} y_{k}
$$

As before, we denote by $P_{i}^{\Re_{\omega, y}}$ a coalition of parties that precede $i$ in the order $\Re_{\omega, y}$. Let

$$
\varphi(i, \omega, y)= \begin{cases}1 & , i \text { is a pivot player to } P_{i}^{\Re_{\omega, y}} \\ 0 & , \text { otherwise }\end{cases}
$$

The Shapley-Owen index is defined by

$$
S O_{i}(y)=\int_{\Omega} f_{\Omega} \varphi(i, \omega, y) d \omega
$$

Denote

$$
\operatorname{Exp}\left(\frac{S O_{i}(Y)}{x_{i}(Y)}, n\right)=\int_{D^{n}} f\left(y_{1}\right) \ldots f\left(y_{n}\right) \frac{S O_{i}(y)}{x_{i}(y)} d y
$$

The following figures summarize Monte-Carlo simulations for $d=1$ and $d=2$. For $d=1$ we consider locations of voters and parties distributed once uniformly on a closed interval, then with the standard normal distribution. For $d=2$ we consider locations of voters and parties distributed once uniformly on a closed space, and finally with the multinormal distribution. The simulation shows, that in expectation the ratio of ShapleyOwen index of a party to its size is approximately 1. 


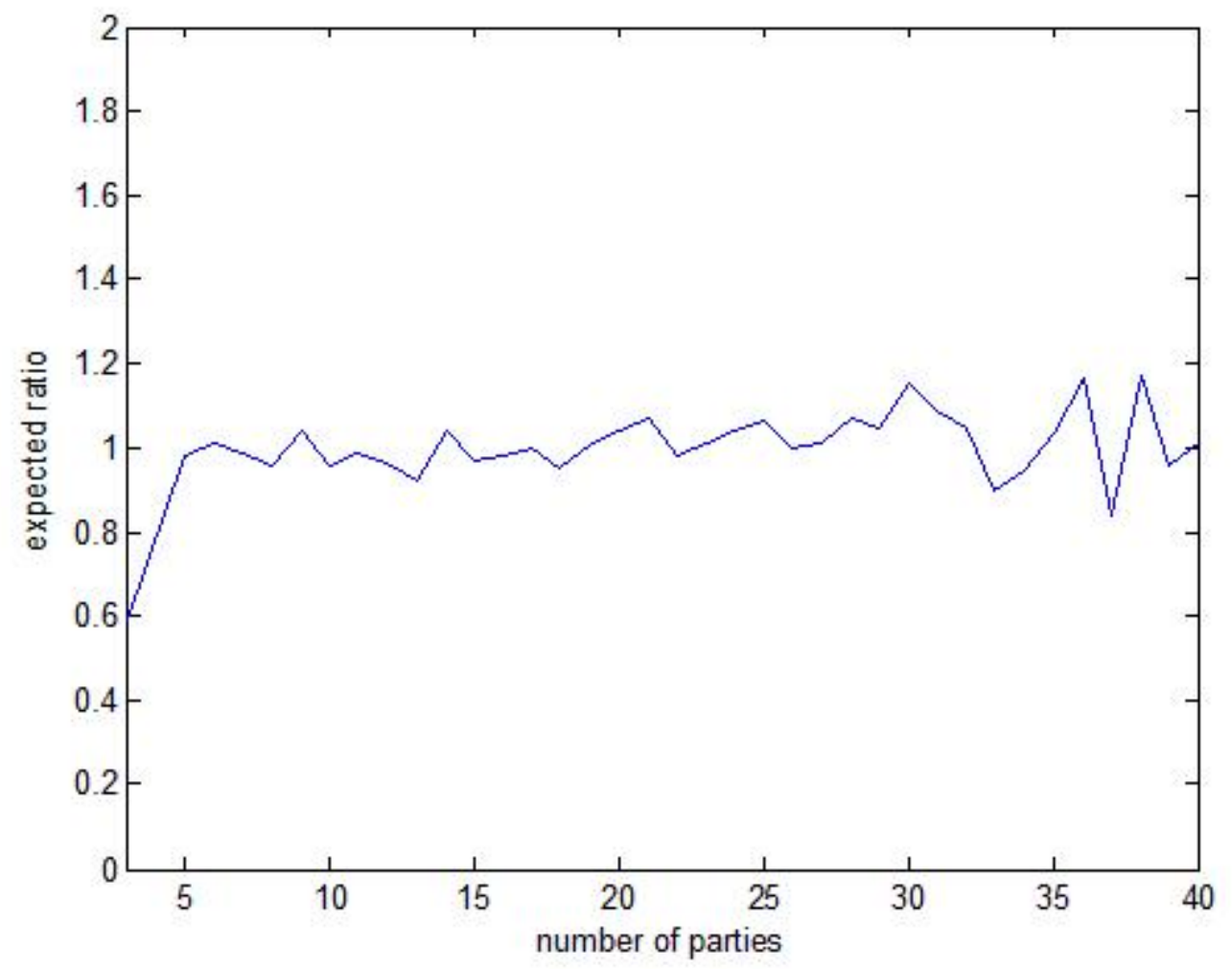

Figure 5: $\operatorname{Exp}\left(\frac{S O_{i}(X)}{X_{i}}, n\right)$ for $q=0.5, d=1$, the uniform distribution 


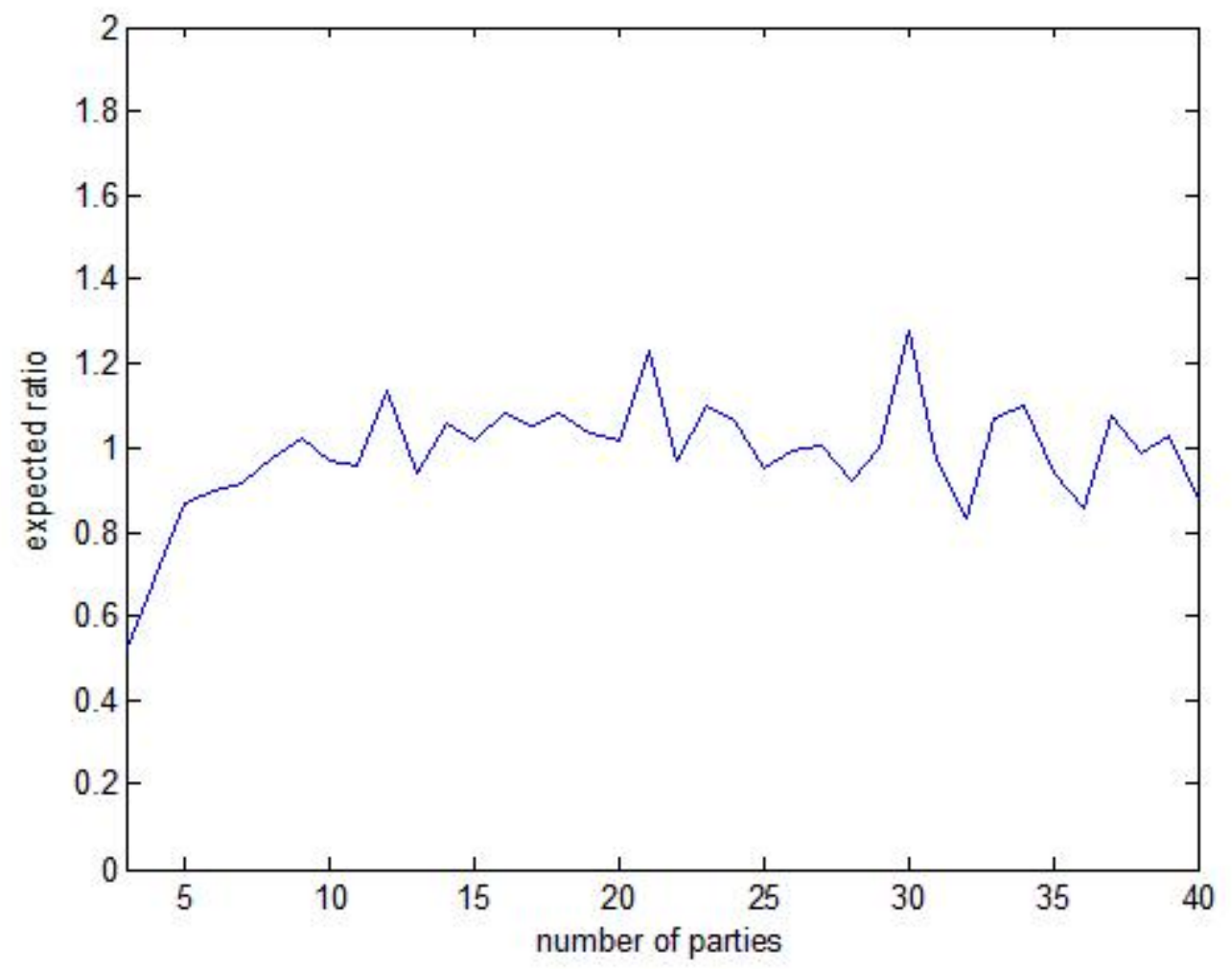

Figure 6: $\operatorname{Exp}\left(\frac{S O_{i}(X)}{X_{i}}, n\right)$ for $q=0.5, d=1$, the normal distribution 


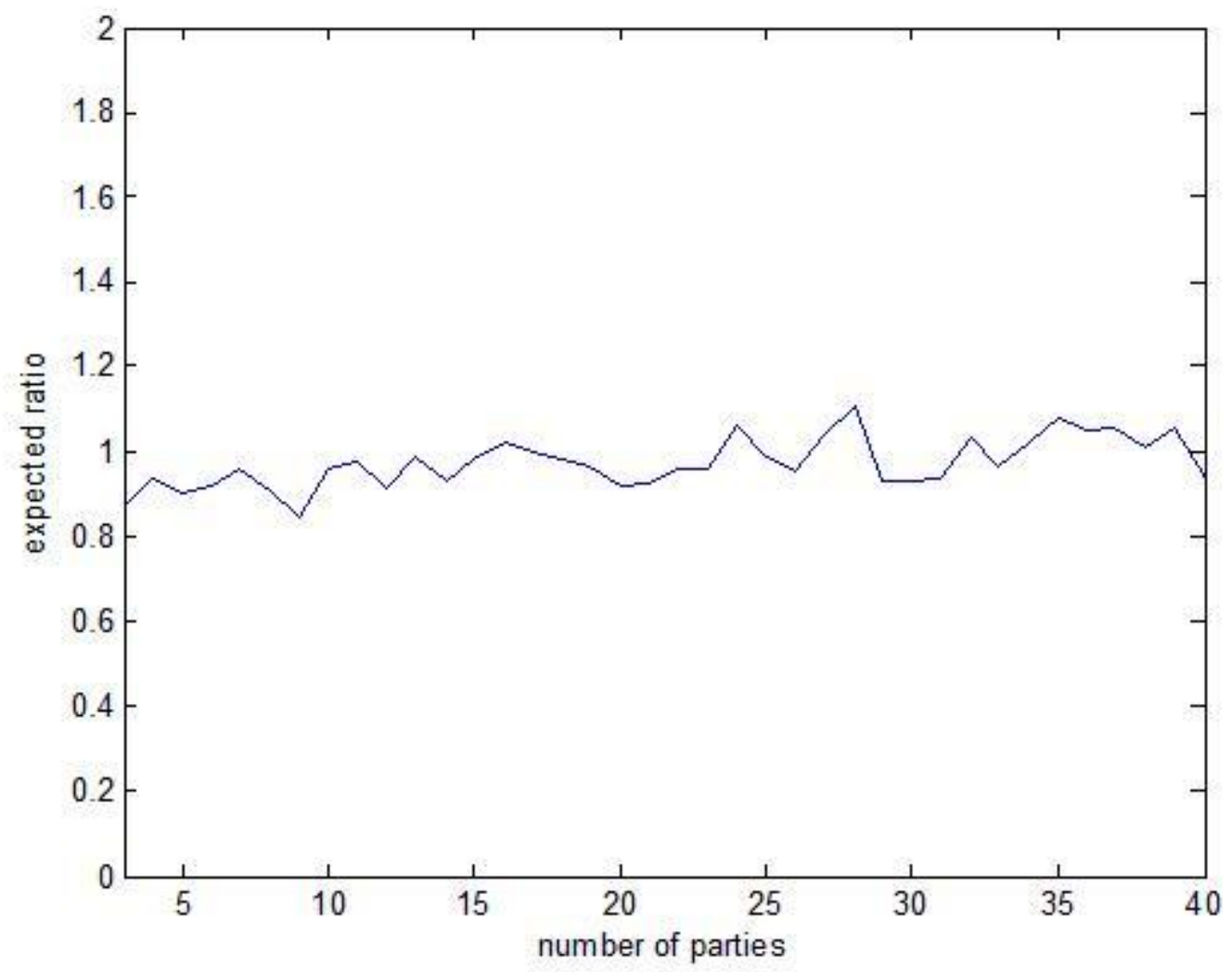

Figure 7: $\operatorname{Exp}\left(\frac{S O_{i}(X)}{X_{i}}, n\right)$ for $q=0.5, d=2$, the uniform distribution 


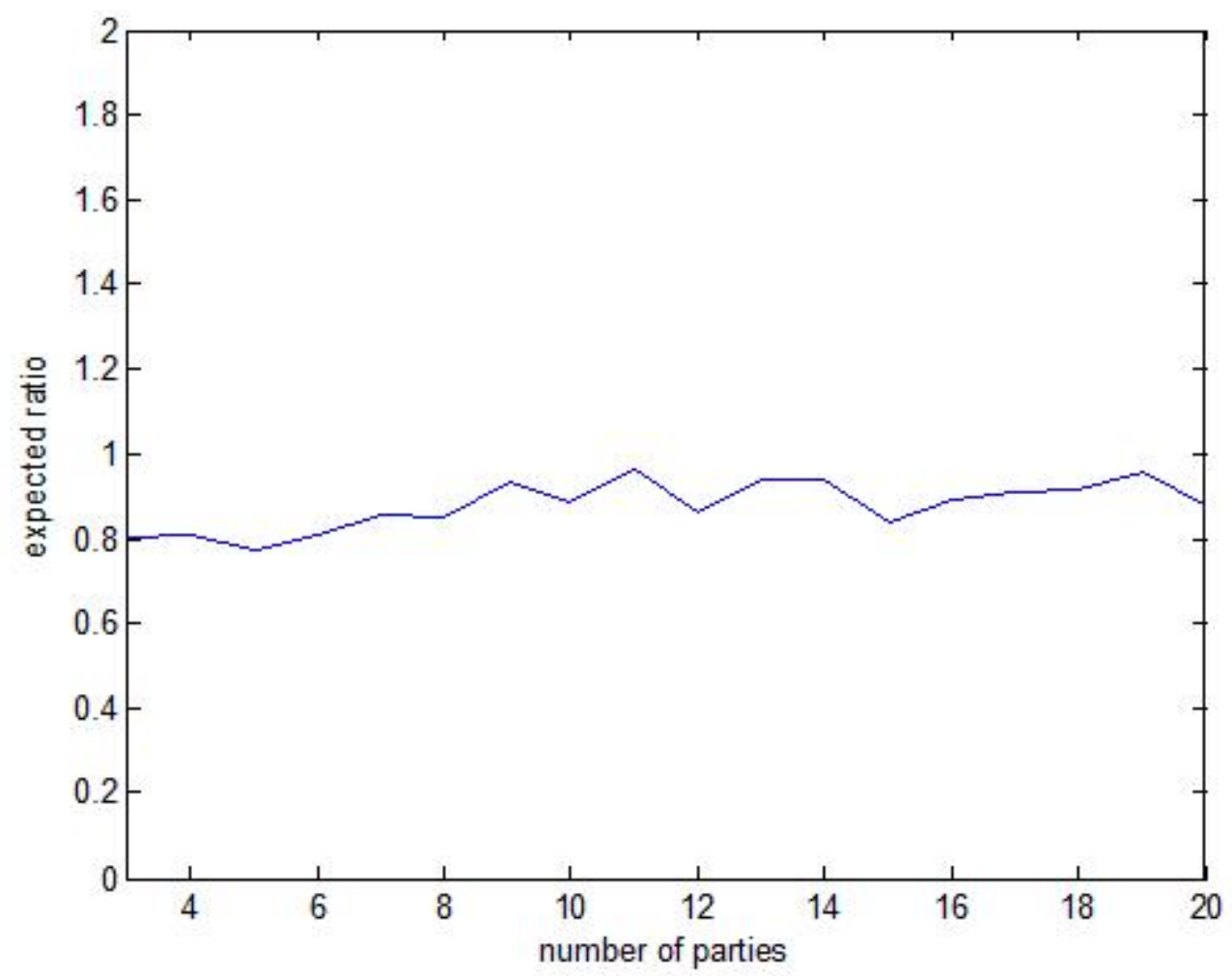

Figure 8: $\operatorname{Exp}\left(\frac{S O_{i}(X)}{X_{i}}, n\right)$ for $q=0.5, d=2$, the multinormal distribution

In the next simulation, we construct a "random" probability distribution $F:[0,1] \rightarrow$ $[0,1], F(0)=0, F(1)=1$. In the first stage we partition the interval $[0,1]$ into small subintervals $\left[x_{0}, x_{1}\right],\left[x_{2}, x_{3}\right], \ldots,\left[x_{m-1}, x_{m}\right], x_{0}=0, x_{m}=1$ of equal size. In the second stage we generate from the uniform distribution a random vector $\left(z_{1}, \ldots, z_{m}\right) \in A_{m}$ and define:

$$
F(x)= \begin{cases}0 & , x=0 \\ \sum_{j=1}^{i} z_{j} & , x=x_{i}, 1 \leq i \leq m \\ \left(x-x_{i}\right)\left(F\left(x_{i+1}\right)-F\left(x_{i}\right)\right) /\left(x_{i+1}-x_{i}\right) & , x_{i}<x<x_{i+1}, 1 \leq i \leq m-1\end{cases}
$$

For each $F$ we calculate $E(n)=\operatorname{Exp}\left(\frac{S O_{1}(X)}{X_{1}}, n\right)$. We repeat the process 1000 times and take the average $\bar{E}_{1000}(n)$ of the 1000 results of $E(n)$. We show (graphically) that $\bar{E}_{1000}(n)$ is close to 1 . 


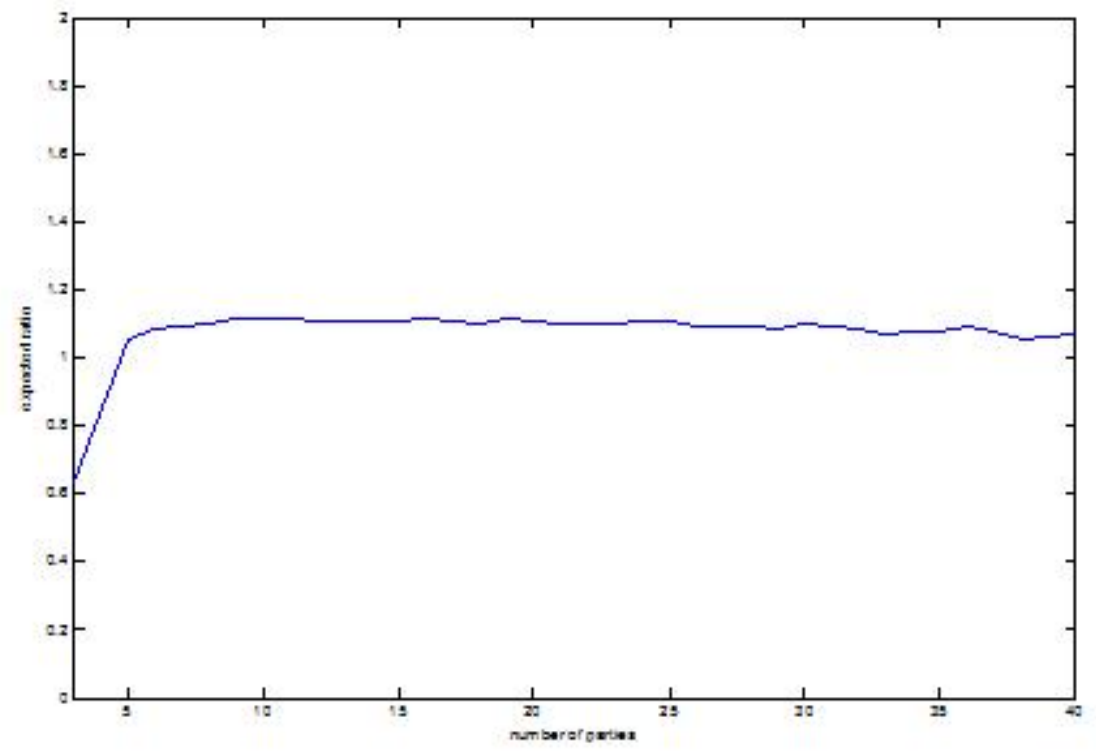

Figure 9: The random process, $q=0.5, d=1$ and $\bar{E}_{1000}(n)$

\section{References}

J.F. Banzhaf. Weighted voting doesn't work: A mathematical analysis. Rutgers L. Rev., 19:317-343, 1964.

J.F. Banzhaf. One man, 3.312 votes: a mathematical analysis of the Electoral College. Vill. L. Rev., 13:304-332, 1968.

P. Dubey. On the uniqueness of the Shapley value. International Journal of Game Theory, 4(3):131-139, 1975.

P. Dubey and L.S. Shapley. Mathematical properties of the Banzhaf power index. Mathematics of Operations Research, pages 99-131, 1979.

P. Dubey, E. Einy, and O. Haimanko. Compound voting and the Banzhaf index. Games and Economic Behavior, 51(1):20-30, 2005. 
W. Feller. An introduction to probability theory and its applications. Vol. II. John Wiley and Sons, New York, 1971.

D.S. Felsenthal and M. Machover. The measurement of voting power: theory and practice, problems and paradoxes. Edward Elgar Cheltenham, UK, 1998.

D.S. Felsenthal and M. Machover. Voting power measurement: a story of misreinvention. Social choice and welfare, 25(2):485-506, 2005.

H. Hotelling. Stability in competition. The economic journal, 39(153):41-57, 1929.

Galen A. Irwin. The Dutch parliamentary election of 1998. Electoral Studies, 18(2):271 $-276,1999$.

MV Jambunathan. Some properties of Beta and Gamma distributions. The annals of mathematical statistics, 25(2):401-405, 1954.

E. Lehrer. An axiomatization of the Banzhaf value. International Journal of Game Theory, 17:89-99, 1988.

Paul Lucardie. The Netherlands. European Journal of Political Research, 42(7-8):10291036,2003

Paul Lucardie. The Netherlands. European Journal of Political Research, 46(7-8):1041$1048,2007$.

Paul Lucardie and Gerrit Voerman. The Netherlands. European Journal of Political Research, 28(3-4):427-436, 1995.

Paul Lucardie and Gerrit Voerman. The Netherlands. European Journal of Political Research, 43(7-8):1084-1092, 2004.

Paul Lucardie and Gerrit Voerman. The Netherlands. European Journal of Political Research, 50(7-8):1070-1076, 2011. 
T.T. Mackie and R. Rose. The international almanac of electoral history, 3rd ed. Macmillan Press Ltd., 1991.

R. Nozick. Weighted voting and'one-man, one-vote'. Representation, ed.Pennock and Chapman, pages 217-225, 1968.

H. Nurmi. The problem of the right distribution of voting power. Power, voting and voting power, ed. Holler, pages 203-212, 1981.

G. Owen. Political games. Naval Research Logistics Quarterly, 18(3):345-355, 1971.

G. Owen. Evaluation of a presidential election game. The American Political Science Review, 69(3):947-953, 1975.

D. Rae, V. Hanby, and J. Loosemore. Thresholds of representation and thresholds of exclusion. Comparative political studies, 3(4):479-488, 1971.

N.Z. Shapiro and L.S. Shapley. Values of large games, i: a limit theorem. Mathematics of Operations Research, pages 1-9, 1978.

L.S. Shapley. A value for n-person games. Contributions to the Theory of Games, ed. Kuhn and Tucker, 2:31-40, 1953.

L.S. Shapley. Values of games with infinitely many players. RAND Corporation, 1961.

L.S. Shapley. A comparison of power indices and a nonsymmetric generalization. $R A N D$ Corporation, 1977.

L.S. Shapley and M. Shubik. A method for evaluating the distribution of power in a committee system. The American Political Science Review, 48(3):787-792, 1954.

P.D. Straffin. Power and stability in politics. Handbook of game theory with economic applications, ed. Aumann and Hart, 2:1127-1151, 1994.

Cees Van Der Eijk. The Netherlands. Electoral Studies, 8(3):305 - 312, 1989. 
H.P. Young. Monotonic solutions of cooperative games. International Journal of Game Theory, 14(2):65-72, 1985. 